\title{
Sentence Context Modulates the Neighborhood Frequency Effect in Chinese Reading: Evidence from Eye Movements
}

\author{
Panpan Yao ${ }^{1}$, Timothy J. Slattery ${ }^{2}$ and Xingshan $\mathrm{Li}^{1}$ \\ ${ }^{1}$ CAS Key Laboratory of Behavioral Science, \\ Institute of Psychology, Beijing, China \\ ${ }^{2}$ Bournemouth University
}

Author Note

This research was supported by a grant from the National Natural Science Foundation of China (31970992), and was also jointly funded by the National Natural Science Foundation of China (NSFC) and the German Research Foundation (DFG) in Project Crossmodal Learning, NSFC 61621136008/DFG TRR-169. All the data and analysis codes in R used in the current study can be found at DOI 10.17605/OSF.IO/54DJ2.

Correspondence should be addressed to Xingshan Li, 16 Lincui Road, Key Laboratory of Behavioral Science, Institute of Psychology, Chinese Academy of Sciences, Beijing, China. Email: lixs@psych.ac.cn. 


\begin{abstract}
In the current study, we conducted two eye-tracking reading experiments to explore whether sentence context can influence neighbor effects in word recognition during Chinese reading. Chinese readers read sentences in which the targets' orthographic neighbors were either plausible or implausible with the pre-target context. The results revealed that the neighbor effect was influenced by context: the context in the biased condition (where only targets but not neighbors can fit in the pre-target context) evoked a significantly weaker inhibitory neighbor effect than in the neutral condition (where both targets and neighbors can fit in the pre-target context). These results indicate that contextual information can be used to modulate neighbor effects during on-line sentence reading in Chinese.
\end{abstract}

Keywords: Chinese reading, context effect, eye-tracking, neighborhood frequency effect, word recognition 


\section{Sentence Context Modulates the Neighborhood Frequency Effect in Chinese Reading: Evidence from Eye Movements}

In natural reading, words are always embedded in sentences instead of occurring in isolation. Thus apart from lexical features such as word frequency (Rayner \& Duffy, 1986; Staub et al., 2010) and orthographic neighbors (Andrews, 1989, 1997; Carreiras et al., 1997; Pollatsek et al., 1999), the context in which the word is embedded also influences lexical processing. To be specific, contextually inappropriate/implausible words are associated with processing difficulties (Cole \& Perfetti, 1980; Foss \& Blank, 1980; Goy et al., 2013; Marslen-Wilson, 1985; Rayner et al., 2004; Warren \& McConnell, 2007). However, how context interacts with lexical features, especially orthographic neighbors, is rarely investigated. The current research aimed to explore how sentence context modulates orthographic neighbor effects in Chinese reading.

Orthographic neighbors in alphabetic languages are words that can be created by changing one letter of the target word while preserving the letters at other positions (Coltheart et al., 1977). They are activated by the visual input of the target word and have been hypothesized to compete with the target through a lateral inhibitory connection at the lexical level (the Interactive Activation Model, henceforth IAM, McClelland \& Rumelhart, 1981; Rumelhart \& McClelland, 1982). The degree of this lateral competition is a function of the relative frequency of the target and its neighbors. Neighbors with higher frequency will compete more actively and thus inhibit the activation of lower frequency neighbors, which is called the inhibitory 
Neighborhood Frequency Effect (Grainger et al., 1989). Previous studies found that words with higher frequency neighbors are harder to recognize and process than words without higher frequency neighbors in both the lexical decision task (Grainger, 1990; Grainger \& Jacobs, 1996; Grainger \& Segui, 1990) and sentence reading task (Perea \& Pollatsek, 1998; but see Sears et al., 2006). Furthermore, the more higher frequency neighbors a word has, the greater the inhibitory effects are (Pollatsek et al., 1999).

Using eye-tracking, Slattery (2009) investigated whether the activation of orthographic neighbors influences word processing, and whether context modulates this effect. Words with higher frequency neighbors were target words, and words without higher frequency neighbors were control words which were matched to target words on many lexical properties (e.g., frequency, word length). There were two types of sentences: in neutral sentences, control words, target words and the targets' higher frequency neighbors were plausible given pre-target text; while in biased sentences, only control and target words were plausible, but the targets' higher frequency neighbors were implausible. Participants were required to silently read the sentences for comprehension. The results revealed a stronger inhibitory neighbor effect (target words were associated with significantly longer second pass times and total reading times than control words) in the neutral condition than the biased condition, indicating that contextual information can mediate the neighbor effect. Slattery argued that in the biased sentences, the higher frequency neighbors were eliminated from the lateral competition since they were inappropriate/implausible given the pre-target context, 
thereby reducing or eliminating the inhibitory effects on target words. Similar results have been reported by Johnson (2009) which investigated context's influence on transposed-letter neighbor effects and Gregg and Inhoff (2016) which investigated context's influence on misidentifying target words as their neighbors.

The above mentioned studies were designed to investigate the effect of context on the neighbor effect in English reading. It is unclear whether context can modulate the neighbor effect in Chinese, which is a non-alphabetic writing system. The definition of orthographic neighbor in Chinese is different from that in alphabetic languages. Orthographic neighbors in Chinese are defined from two aspects: character level and word level. On the character level, characters sharing the same radical (Li et al., 2011; Wang et al., 2014; Wu \& Chen, 2003; Yang \& Wu, 2014) or sharing around two thirds of strokes (Dong et al., 2015) are defined as orthographic neighbors. Previous studies found an inhibitory neighbor effect on the character level (Li et al., 2011; Wu \& Chen, 2003; Yang \& Wu, 2014). On the word level, orthographic neighbors are defined as words which only have one constituent character difference while share all the other characters at their positions (Huang et al., 2006; Tsai et al., 2006). For example, “花园” (/hualyuan2/, garden) and “花店” (/hualdian4/, flower store) share the constituent “花” (/hua1/, flower) in the first position and differ with each other in the second constituent. These two words are orthographic neighbors and can be called first character orthographic neighbors since they share the first constituent and differ with each other on the second constituent ${ }^{1}$. On the other hand,

\footnotetext{
${ }^{1}$ Note that in the current study, orthographic neighbors are defined as two-character nouns which share
} 
“花园” (/hua1yuan2/, garden) and “公园” (/gong1yuan2/, park), which share the second constituent “园” (/yuan2/, an area of land for a certain purpose), are second character orthographic neighbors. Previous studies found in two-character words, the leading character has a dominant influence on word recognition, and there is an inhibitory neighbor effect on the word level (Huang et al. 2006; Li et al., 2017). In the current research, we focused on word level first character orthographic neighbors of two-character nouns in Mandarin Chinese. Since the shared components among neighbors are characters in Chinese instead of letters in alphabetic languages such as English, the basic assumption of a discrete input-letter level incorporated in many word recognition models may not be applicable to Chinese. Thus, it is important to explore how context influences the activation of neighbors in Chinese reading. The current study offers a valuable comparison with related studies in alphabetic languages.

It should be noted that so far no reading model can account for neighbor effect and context effect simultaneously. First, the activation of neighbors was rarely embodied by computational models of eye movement control in reading. For example, the E-Z Reader Model (Pollatsek et al., 2006; Rayner et al., 2004; Reichle et al., 1998; Reichle et al., 1999; Reichle et al., 2007; Reichle et al., 2009), and SWIFT Model (Engbert et al., 2005; Laubrock et al., 2006; Richter et al., 2006), and

\footnotetext{
the same positional-specific character, independent of the meanings conveyed by the character. For example, the character “花” (/hua1/, flower) in “花园” (/hualyuan2/, garden) conveys a "flower” meaning, while it in “花费” (/hual fei4/, expense) conveys a "to spend" meaning. Though such characters have multiple meanings which give them multiple morphological families but they only have one set of orthographic neighbors. We focused on orthographic similarity among words only in the current study.
} 
Glenmore Model (Reilly \& Radach, 2003, 2006) do not take the activation of neighbor words into consideration and cannot account for the observed neighbor effects. Second, for some models which considered the activation of orthographic neighbors, they cannot account for the context's modulation on neighbor effects. For instance, the recent OB1-Reader Model (Snell et al., 2018) does allow for some activation of word neighbors in alphabetic languages via open bigram coding. However, OB1-reader would not be capable of capturing the potential interactions between context and word neighbors as it contains only one contextual parameter which codes the predictability of the target word (but not its neighbors). Similarly, a recently developed Chinese Reading Model (henceforth CRM, Li \& Pollatsek, 2020) which is built based on the IAM proposed that in Chinese sentence reading, all the characters in the perceptual span are activated, and all the possible words formed by these characters (e.g., orthographic neighbors) and highly similar characters are activated and compete with each other for recognition. CRM successfully simulated some important findings in Chinese reading, and can potentially account for the neighbor effects. However, it did not give explicit predictions regarding how sentence context influences neighbor effects in Chinese reading since only the predictability of the target (but not its neighbors) was embedded as a sole contextual parameter. Therefore, we speculate that the findings in the current study will contribute to the development of existing reading models.

We designed two eye-tracking experiments to investigate whether sentence context can modulate the neighbor effect during Chinese reading. Different from 
Slattery (2009) which measured the neighbor effect by comparing target words (words with higher frequency neighbors) with control words (words without higher frequency neighbors) in different contextual conditions (neutral vs. biased), we manipulated sentence contextual constraints so that the plausibility of targets' neighbors in prior sentential fragments varied in different contextual conditions and directly compared the processing of the same target words in these conditions. By doing so we could eliminate the possible impact of lexical variables (e.g., neighbor size between target words and control words). In addition, in Slattery's (2009) study, all target words were the lower frequency member of a pair of orthographic neighbors. Here we explore the neighbor effect from both higher frequency neighbors (Experiment 1) and lower frequency neighbors (Experiment 2).

In Experiment 1, the plausibility of targets' highest frequency neighbors in prior sentential fragments varied. In the neutral condition, the targets' highest frequency neighbor and many higher frequency neighbors could fit in the prior context; while in the biased condition, only a few higher frequency neighbors could fit in prior context, but not the targets' highest frequency neighbor. If context modulates the activation of neighbors, the manipulation of contextual constraints would affect the processing of target words. In the neutral condition, target words and their higher frequency neighbors which fit with the prior context would be activated and compete for access, resulting in slow processing (i.e., an inhibitory neighbor effect). On the other hand, the biased condition would have fewer activated higher frequency neighbors competing for access (some of higher frequency neighbors would be deactivated due 
to their implausibility in pre-target context) thereby reducing or eliminating the inhibitory neighbor effect. Consequently, the processing of target words should be harder in the neutral than in the biased condition. However, if context does not modulate the activation of neighbors, the processing of target words should not significantly differ between the neutral and biased conditions.

In Experiment 2, we looked into whether having lower frequency neighbors would affect the processing of higher frequency target words in Chinese, and whether context would modulate this possible effect. The IAM predicts a frequency-related asymmetry of neighbor effects. Higher frequency neighbors have higher activation resting levels than lower frequency neighbors, resulting in greater lateral competition. A frequency-related asymmetry has been observed by several previous studies of alphabetic reading (Gregg \& Inhoff, 2016; Pollatsek et al., 1999; Williams et al., 2006). For example, Gregg and Inhoff (2016) found that lower frequency targets were often misidentified as higher frequency neighbors, but not vice versa. Furthermore, Williams et al. (2006) showed in two experiments with boundary technique that higher frequency neighbor previews resulted in as much preview benefit as the target word itself while lower frequency neighbor previews resulted in no more preview benefit than non-word controls. However, Johnson (2009) found a different pattern with transposed-letter neighbors: interference effects were present regardless of whether the target word was the lower or higher frequency member of the transposedletter neighbor pair. Also, Paterson et al. (2009) reported that reading a target word's orthographic neighbor earlier in a sentence inhibits the later reading of the target, 
regardless of whether the neighbor was a higher or lower frequency member. Therefore, while the body of evidence suggests a frequency asymmetry in the neighbor effect, not all studies have found this asymmetry. Experiment 2 explored the possibility of this frequency asymmetry for Chinese reading. Experiment 2 contrasts directly to Experiment 1 in that the highest frequency neighbors from Experiment 1 were treated as target words in Experiment 2, while the targets from Experiment 1 were treated as lower frequency neighbors in Experiment 2.

\section{Experiment 1}

In Experiment 1, to investigate how sentence context modulates the neighbor effect in Chinese sentence reading, we manipulated the contextual constraints of sentences so that the plausibility of target words' higher frequency orthographic neighbors varied given the prior context.

\section{Participants}

Sixty-four students (47 female, age range 18-40 years) from universities around the Institute of Psychology, Chinese Academy of Science participated in the experiment. All of them were native speakers of Mandarin Chinese and had normal or corrected-to-normal vision. Four participants were excluded from data analysis due to their low accuracy on comprehension questions $(<85 \%)$.

\section{Materials}

In the current study, we used 40 two-character words as target words. Targets had at least one higher frequency orthographic neighbor (which shared the first character 
with the target, but had a different second character). Descriptive statistics of these words, including the mean neighborhood size of the first character, the mean stroke number of the first and the second character, the mean frequency and the mean ranking based on frequency in the neighbor group are summarized in Table 1.

\section{Table 1}

The descriptive statistics of target words in Experiment $1 \&$ Experiment 2

Targets in Targets in

Experiment $1 \quad$ Experiment 2
42.38

6.43

8.40

7.65
42.38

6.58

327.75

1

Target words were embedded in two conditions of sentences: in the neutral condition, both target words and many of higher frequency neighbors (including the highest frequency neighbors) fit in the pre-target context; in the biased condition, only target words fit in the sentence, but not highest frequency neighbors (nor many other higher frequency neighbors). Therefore, the number of higher frequency neighbors which were plausible in prior context varied between the different context conditions. The plausibility of target words and their highest frequency neighbors in the sentences was assessed by 40 native Mandarin speakers (who did not participate in Experiment 
1). They were given the sentence fragments up to (including) the targets or their highest frequency neighbors, and were asked to judge whether the last word was grammatical and acceptable to occur in the context by using a 5-point scale, in which 1 stands for ungrammatical and unacceptable, while 5 stands for grammatical and acceptable. The mean scores for target words in the neutral and biased conditions were 4.72 and 4.82 , respectively $(t(78)=-1.62, p=.11)$, and the mean scores for the highest frequency neighbors were 4.75 and 1.45 , respectively $(t(78)=48.12, p$ $<.001)$. Furthermore, an on-line survey was conducted with ten native Mandarin speakers (who did not participate in Experiment 1 nor the other norming tests) to assess which higher frequency neighbors could fit with the pre-target context. For each pre-target sentential fragment, all the higher frequency neighbors of the target word were laid out, among which participants were asked to choose the words (as many as they want, zero or multiple) that were plausible and appropriate continuations of these sentential fragments. If a word was chosen by more than four participants, it was treated as a plausible continuation of the context. There were significantly more higher frequency neighbors which could fit in the prior context in the neutral condition $(M=4.60)$ than in the biased condition $(M=1.20), t(78)=3.58$, $p<.001$.

There were 80 sentences in total (40 sentences in each condition). The lengths of the sentences varied from 9 to 24 characters. The average positions of target words in sentences were matched between neutral and biased conditions, $p \mathrm{~s}>.9$. All of these sentences were normed by ten native Mandarin speakers to ensure that they were 
grammatical and acceptable. Due to the limited number of available materials and participants, all the 80 sentences were presented to each participant, plus 50 fillers which did not contain either the target words or their higher frequency neighbors. In other words, each participant read both neutral and biased trials of a certain target word. Neutral and biased trials were intermixedly presented to participants with the presentation order counter-balanced among participants, and the number of trials inserted between neutral and biased trials of the same target word was fixed at nine. Examples of materials are illustrated in Table 2. None of the higher frequency neighbors were presented in the experiment.

\section{Table 2}

Material examples in Experiment 1

\begin{tabular}{|c|c|}
\hline Condition & Sentence \\
\hline \multirow{2}{*}{ Neutral } & 那个戴眼镜的就是老师（老人）的女儿。 \\
\hline & The one with the glasses is the daughter of the teacher/old man. \\
\hline \multirow{2}{*}{ Biased } & 小明最害怕班主任老师（*老人）来家访了。 \\
\hline & XiaoMing is scared of being visited by his class teacher/*old man. \\
\hline
\end{tabular}

Note. 老师 (/lao3shi1/, teacher) is the target word, and 老人 (/lao3ren2/, old man) is its highest frequency neighbor. In the neutral condition, both 老师 and 老人 can fit in the context prior to the target, while in the biased condition, only 老师 can fit the prior context, but not its highest frequency neighbor 老人. In Experiment 1, highest frequency neighbor 老人 was never presented.

\section{Apparatus}

Participants' eye movements were recorded using Eyelink 1000 (SR Research Inc., Toronto, Canada) eye tracker with a sampling rate of $1,000 \mathrm{~Hz}$. Sentences were 
presented in one line at the middle of a 21 -inch cathode ray tube (CRT) monitor.

Participants were seated $57 \mathrm{~cm}$ away from the monitor. Following a 3-point calibration and validation, the gaze-position error was less than $0.5^{\circ}$. Eye movements were recorded from the right eye, but viewing was binocular.

\section{Procedure}

Participants were tested individually. The experiment started with a brief instruction and a standard horizontal three-point grid calibration and validation. Then, five practice trials were run to ensure that the participants understood the task and were familiar with the apparatus. Critical experimental trials were run after the practice trials. Participants were required to read sentences silently, each of which was presented in one line in the middle of the screen. At the beginning of each trial, a drift check was conducted. Each sentence appeared after participants fixated on a character-sized box at the location of the first character of each sentence. After reading each sentence, participants were asked to press a certain button so that the original sentence disappeared and was replaced by a meaning-related question. Participants were asked to answer the question by pressing corresponding buttons. The entire experiment lasted about 20 mins.

\section{Results}

Four eye-movement indexes on target words were analysed. First fixation duration (FFD) is the duration of the first fixation on a target word. Gaze duration (GD) is the sum of the fixation durations before the eyes first move out of a target. 
Total reading time (TT) is the sum of the durations of all fixations on a target.

Regression-in probability is the proportion of trials where readers looked back from the right after they first passed this target. The mean response accuracy for the 60 participants was 97\%. We chose only the correctly answered trials for the final analysis. Fixations shorter than $80 \mathrm{~ms}$ or longer than 1,000 ms were removed (less than $1 \%$ of total fixations).

A linear mixed effects (LME) model (Baayen et al., 2008) was used on logtransformed FFD, GD and TT, which yield the same pattern of statistical significance as the analysis based on raw data. In the interest of transparency of effect sizes, we report the analysis of the untransformed data here. The generalized LME model was used on regression-in probability. In each LME/GLME model, the context condition (neutral vs. Biased, which was coded as $.5,-.5$ respectively), and the presentation order of each pair of items (for each target word, the neutral trial was either firstpresented, i.e., before the biased trial, or second-presented, i.e., after the biased trial, which was coded as $-.5, .5$ respectively) was added as fixed effects, specifying participants and items as random effects, including intercepts and slopes. The random effects structures of the models were trimmed down when the models failed to converge based on Bates et al. (2015). To directly test the context effect in first- or second-presented trials, following Schad et al. (2020), we used the following three customized contrasts: (1) a contrast that tests the main effect of presentation order (first-presented vs. second-presented); (2) a contrast that tests the context effect for the first-presented trials; and (3) a contrast that tests the context effect for the second- 
presented trials. The statistical procedure was conducted using the lmer program

(lme4 package; Bates et al., 2011) in $R$ (version 3.5.1; R Core Team 2018). The means and SEs for each index in each condition are summarized in Table 3. We report the statistical analysis results by focusing on beta, $S E, t / z$ and $p$ values, which are summarized in Table 4.

\section{Table 3}

Summary of the means and SEs (in parentheses) for each index in each condition with presentation order in Experiment 1

\begin{tabular}{lcccc}
\hline \multirow{2}{*}{ Eye-Movement measures } & \multicolumn{2}{c}{ First-presented } & \multicolumn{2}{c}{ Second-presented } \\
\cline { 2 - 5 } & Neutral & Biased & Neutral & Biased \\
\hline First fixation duration (ms) & $264(7.13)$ & $239(6.62)$ & $249(7.53)$ & $245(5.10)$ \\
Gaze duration (ms) & $295(8.59)$ & $262(7.93)$ & $277(10.23)$ & $272(7.26)$ \\
Total reading time (ms) & $449(19.22)$ & $363(13.83)$ & $396(19.57)$ & $379(18.53)$ \\
Regression-in probability & $0.37(0.02)$ & $0.26(0.02)$ & $0.32(0.02)$ & $0.28(0.03)$ \\
\hline
\end{tabular}

\section{Table 4}

Summary of the LME/GLME analysis results in Experiment 1 (Dependent Variables $\sim$ order/context)

\begin{tabular}{|c|c|c|c|c|}
\hline & Estimate & SE & $t / z$ & $p$ \\
\hline \multicolumn{5}{|c|}{ First fixation duration } \\
\hline (Intercept) & 249.55 & 5.06 & 49.29 & $<.001 * * *$ \\
\hline Order & -4.35 & 3.21 & -1.35 & .18 \\
\hline Order $1^{\text {st }}:$ context & 24.09 & 10.69 & 2.26 & $.03 *$ \\
\hline Order $2^{\text {nd: }}$ context & 3.26 & 10.56 & 0.31 & .76 \\
\hline \multicolumn{5}{|l|}{ Gaze duration } \\
\hline (Intercept) & 276.93 & 6.39 & 43.35 & $<.001 * * *$ \\
\hline Order & -3.08 & 4.70 & -0.66 & .51 \\
\hline Order $1^{\text {st }}:$ context & 30.90 & 13.36 & 2.31 & $.02 *$ \\
\hline Order $2^{\text {nd: }}$ context & 4.45 & 13.86 & 0.32 & .75 \\
\hline \multicolumn{5}{|l|}{ Total reading time } \\
\hline (Intercept) & 396.61 & 13.37 & 29.66 & $<.001 * * *$ \\
\hline Order & -17.75 & 8.16 & -2.18 & $.03 *$ \\
\hline Order $1^{\text {st }}:$ context & 86.77 & 27.68 & 3.13 & $<.01 * *$ \\
\hline
\end{tabular}




\begin{tabular}{lcccc}
\hline Order $2^{\text {nd. }}$ : context & 17.51 & 28.24 & 0.62 & .54 \\
$\begin{array}{l}\text { Regression-in probability } \\
\text { (Intercept) }\end{array}$ & -0.87 & 0.07 & -12.90 & $<.001^{* * *}$ \\
Order & -0.06 & 0.09 & -0.70 & .49 \\
${\text { Order } 1^{\text {st }}: \text { context }}_{\text {Order } 2^{\text {nd. }} \text { context }}$ & 0.52 & 0.16 & 3.34 & $<.001^{* * *}$ \\
\hline
\end{tabular}

The context effect was significant for first-presented trials in all the four eye movement measures, with longer fixations and more regressions on targets in the neutral condition than in the biased condition. This is consistent with previous studies in alphabetic reading (Slattery, 2009). The inhibitory effects indexed by longer fixations and higher regression-in probability were treated as evidence of the interference from higher frequency neighbors on target word recognition (Acha \& Perea, 2008; Perea \& Pollatsek, 1998; Pollatsek et al, 1999). Thus the observed pattern in first-presented trials indicates that there was a stronger inhibitory neighbor effect in the neutral condition than in the biased condition, suggesting that in Chinese sentence reading, context can influence the activation of lexical candidates during word recognition.

Regarding the second-presented trails, there was no significant context effect. In addition, a significant effect of presentation order was observed in TT, with longer reading times on targets in first-presented trials than second-presented trials. We speculate that participants' behaviour on second-presented trials was affected by practice or the repetition of target words. To be specific, we postulate that the repetitive occurrences of targets changed the activation levels of representations, and consequently led to faster processing (Kolers \& Ostry, 1974; Raney, 2003; 
Scarborough et al., 1977).

\section{Experiment 2}

In Experiment 2, to investigate whether having lower frequency neighbors would affect the processing of higher frequency neighbors in Chinese reading, and whether context would modulate this possible effect, we manipulated the contextual constraints of sentences so that the plausibility of target words' lower frequency orthographic neighbors varied given the prior context.

The IAM predicts that lower frequency targets would suffer inhibitory effects from higher frequency neighbors, while higher frequency targets would be largely immune to the inhibitory effects from lower frequency neighbors. Thus, no context effect would be expected in Experiment 2. This is because in either the neutral or biased condition, lower frequency neighbors would not affect the processing of the highest frequency targets. On the other hand, if the inhibitory neighbor effect is frequency-independent, as reported by Johnson (2009) and Paterson et al. (2009), processing difficulties of target words would be expected in the neutral condition but not in the biased condition.

\section{Participants}

Sixty-one students (40 female, age range 18-40 years) from universities around Institute of Psychology, Chinese Academy of Science participated in the experiment (none of them participated in Experiment 1 or the norming tests). All of them are native speakers of Mandarin Chinese and have normal or corrected-to-normal vision. 
One participant was excluded from data analysis due to his/her low accuracy on comprehension questions $(<85 \%)$.

\section{Materials}

There were 40 target words (which were the highest frequency neighbors in Experiment 1). The basic features of these words are summarized in Table 1. Each word was embedded in two sentence frames in which the plausibility of the targets' lower frequency neighbors varied. In the neutral sentences, the target word and many of its lower frequency neighbors fit with the pre-target context; in the biased sentences, only the target word and few of its lower frequency neighbors fit with the pre-target context. The plausibility of target words in these sentences was normed by 20 native Mandarin speakers in a similar way (using a 5-point scale) in Experiment 1. The mean scores for target words in the neutral and biased conditions were 4.75 and 4.74 respectively $(t(78)=.07, p=.94)$. In addition, the plausibility of the lower frequency neighbors of each target was normed by ten native Mandarin speakers in a similar fragment completion test as in Experiment 1 . There were significantly more lower frequency neighbors which could fit in the prior context in the neutral condition $(M=8.65)$ than in the biased condition $(M=1.62), t(78)=5.80, p<.001$.

There were 80 experimental sentences in total (40 sentences in each condition). The lengths of the sentences varied from 9 to 24 characters. The average positions of target words in sentences were matched between the neutral and biased conditions, $p \mathrm{~s}>$.9. All these sentences were normed by ten native Mandarin speakers to ensure 
that they were grammatical and acceptable. Each participant read all these 80 sentences plus 50 fillers which did not contain either the target words or their neighbors. The presentation order of neutral and biased trials was counter-balanced among participants, and the number of trials inserted between the neutral and biased trials of the same target word was fixed at nine. Examples of materials are illustrated in Table 5. None of the lower frequency neighbors were presented in the experiment.

\section{Table 5}

Material examples in Experiment 2

\begin{tabular}{ll}
\hline Condition & \multicolumn{1}{c}{ Sentence } \\
\hline \multirow{3}{*}{ Neutral } & $\begin{array}{l}\text { 那个戴眼镜的就是老人 (老师) 合唱团的指挥。 } \\
\text { The one with the glasses is the leader of the chorus of teachers/old } \\
\text { men. } \\
\text { Bu兄弟个为了瓜分老人 (*老师) 留下的遗产不惜反目成仇。 } \\
\text { The two brothers are against with each other for the allocation of the } \\
\text { inheritance left by their old man/*teacher. }\end{array}$ \\
\hline
\end{tabular}

Note. 老人 (/lao3ren2/, old man) is the target word, and 老师 (/lao3shi1/, teacher) is its lower frequency neighbor. In the neutral condition, both 老人 and 老师 can fit in the context prior to the target, while in the biased condition, only 老人 can fit the prior context, but not its lower frequency neighbor 老师. In Experiment 2, lower frequency neighbor 老师 was never presented.

\section{Apparatus}

Same as in Experiment 1.

\section{Procedure}

Same as in Experiment 1.

\section{Results}


Similar to Experiment 1, four eye-movement indexes on target words were analyzed (FFD, GD, TT and regression-in probability). The mean response accuracy for the 60 participants was $96 \%$. Only the correctly answered trials were chosen for the final analysis. Fixations shorter than $80 \mathrm{~ms}$ or longer than $1000 \mathrm{~ms}$ were removed (less than $1 \%$ of total fixations). The means and $S E s$ for each index in each condition are summarized in Table 6 . We report the statistical analysis by focusing on beta, SE, $t / z$ and $p$ values, which are summarized in Table 7.

\section{Table 6}

Summary of means and SEs (in parentheses) for each index in each condition with presentation order in Experiment 2

\begin{tabular}{lcccc}
\hline \multirow{2}{*}{ Eye-Movement Measures } & \multicolumn{2}{c}{ First-presented } & \multicolumn{2}{c}{ Second-presented } \\
\cline { 2 - 5 } & Neutral & Biased & Neutral & Biased \\
\hline First fixation duration (ms) & $250(4.45)$ & $238(5.06)$ & $231(4.26)$ & $240(5.10)$ \\
Gaze duration (ms) & $291(7.64)$ & $255(6.26)$ & $243(4.43)$ & $255(7.14)$ \\
Total reading time (ms) & $443(20.53)$ & $332(14.65)$ & $325(13.00)$ & $343(13.24)$ \\
Regression-in probability & $0.33(0.02)$ & $0.26(0.02)$ & $0.26(0.02)$ & $0.26(0.02)$ \\
\hline
\end{tabular}

\section{Table 7}

Summary of the LME/GLME analysis results in Experiment 2 (Dependent

Variables order/context)

\begin{tabular}{|c|c|c|c|c|}
\hline & Estimate & SE & $t / z$ & $p$ \\
\hline \multicolumn{5}{|c|}{ First fixation duration } \\
\hline (Intercept) & 239.90 & 3.65 & 65.65 & $<.001 * * *$ \\
\hline Order & -8.60 & 3.09 & -2.78 & $.01 * *$ \\
\hline Order $1^{\text {st: }}$ context & 11.59 & 7.92 & 1.47 & .15 \\
\hline Order $2^{\text {nd. }}$ context & -10.60 & 7.95 & -1.33 & .19 \\
\hline \multicolumn{5}{|l|}{ Gaze duration } \\
\hline (Intercept) & 260.88 & 5.25 & 49.74 & $<.001 * * *$ \\
\hline Order & -23.36 & 4.63 & -5.05 & $<.001 * * *$ \\
\hline Order $1^{\text {st: }}$ context & 36.19 & 11.83 & 3.06 & $<.01 * *$ \\
\hline
\end{tabular}




\begin{tabular}{lcccc}
\hline Order $2^{\text {nd: context }}$ & -12.88 & 11.09 & -1.16 & .25 \\
Total reading time & & & & \\
(Intercept) & 360.27 & 11.29 & 31.90 & $<.001^{* * *}$ \\
Order & -52.95 & 9.37 & -5.65 & $<.001^{* * *}$ \\
Order $1^{\text {st. context }}$ & 113.10 & 25.75 & 4.39 & $<.001^{* * *}$ \\
Order $2^{\text {nd: context }}$ & -16.53 & 23.08 & -0.72 & .48 \\
Regression-in probability & -1.03 & 0.07 & -14.49 & $<.001^{* * *}$ \\
(Intercept) & -0.18 & 0.10 & -1.76 & .08. \\
Order & 0.35 & 0.16 & 2.16 & $.03^{*}$ \\
Order $1^{\text {st: context }}$ & -0.01 & 0.18 & -0.05 & .96 \\
Order 2 $2^{\text {nd: context }}$ & & & &
\end{tabular}

The context effect was significant for first-presented trials in GD, TT and regression-in probability, with longer reading times and more regressions on targets in the neutral condition than in the biased condition. This is consistent with the findings in Pollatsek et al. (1999). In their Experiment 3 they found that when equating the number of higher frequency neighbors, increasing the number of lower frequency neighbors produced inhibition of target processing. In the current experiment, there were significantly more plausible lower frequency neighbors in the neutral condition than in the biased condition which led to harder processing of target words. This suggests that in Chinese reading, lower frequency neighbors inhibit target processing, and sentence context can modulate this inhibitory effect: compared to the neutral condition, the prior context in the biased condition prevented or reduced activation of lower frequency neighbors, thereby reducing lexical competition during word recognition.

Regarding to second-presented trails, there was no significant context effect. In addition, a significant effect of presentation order was observed in FFD, GD and TT, with longer reading times on targets in first-presented trials than second-presented 
trials. Similar to Experiment 1, we speculate that participants' behaviors on secondpresented trials were affected by repetition effects.

\section{General Discussion}

In the current research, we conducted two eye-tracking experiments to explore whether context can modulate the neighbor effect in Chinese reading. The plausibility of a target words' higher (Experiment 1) and lower frequency neighbors (Experiment 2) with prior context was varied so that in neutral sentences, target words and their neighbors were contextually appropriate in the pre-target context, while in biased sentences, the number of plausible neighbors was significantly reduced. As indicated by the results in both experiments, participants' processing of second-presented words was affected by the repetition effects and processing strategies, therefore we focus on interpreting the patterns observed in first-presented trials only.

Target words in the first-presented trials were associated with longer fixations and more regressions in the neutral condition than in the biased condition in both experiments. This finding revealed that both higher- and lower-frequency neighbors can inhibit target word processing (Johnson, 2009; Paterson et al., 2009), and sentence context can influence visual word recognition by narrowing the set of lexical candidates that compete for access (Slattery, 2009).

\section{Comparisons Between Experiment 1 and Experiment 2}

We observed inhibitory neighbor effects in the neutral condition compared to the biased condition in both experiments. This inhibitory neighbor effect may be 
explained by lateral competition among activated neighbors, and also by the misidentify-reanalysis procedure (Slattery, 2009). Note that these two are not mutually exclusive explanations. Previous studies found that readers occasionally misidentify the target word as a neighbor (in most cases, a higher frequency neighbor, Coltheart et al., 2001; Davis, 2003; Grainger \& Jacobs, 1994, 1996; Gregg \& Inhoff, 2016). When readers reach the subsequent context where the misidentified-neighbor is anomalous, the misidentification would require readers to reanalyse the target word, resulting in regressions back to target words for re-reading. Furthermore, it is more likely for readers to misread targets as neighbors when the initial processing of these words was shallow (e.g., with comparatively short GD), and the cost of this misidentification in the first-pass processing needs to be paid in the late stage of processing through regressions back to target words. In other words, this misidentifyreanalysis procedure can be indexed by a negative correlation between GD and regressions-in probability. To be specific, compared to targets with long GD, those with short GD represent shallower processing, resulting in greater likelihood of misidentification and re-reading in the late stage.

To test the possibility of this misidentify-reanalyse procedure, we conducted a post hoc analysis to explore the potential effects of GD on subsequent regression-in probability in both experiments for the first-presented trials. A GLME model was built with the centered target GD $(\log )$ and context condition as fixed factors to predict regressions back to targets. The results are summarized in Table 8 and visualized in Figure 1. In Experiment 1, there is a significant negative correlation between GD and 
regression-in probability in the neutral condition, but a null effect in the biased condition. In Experiment 2, there is no significant relation between GD and regression-in probability in either condition.

To further compare the relation between GD and regression-in probability in two experiments directly, we conducted an omnibus test which included experiment as an additional factor. The results are summarized in Table 9. Although the three-factor interaction is marginally significant $(p=0.06)$, the difference of the patterns between Experiment 1 and Experiment 2 is obvious in Figure 1. According to Brysbaert (2019), the finding that the three-way interaction is approaching but not reaching significance is interpreted by the comparatively low power of testing a three-way interaction in a post hoc analysis, instead of the null interaction per se. Thus we argue that the relations between GD and regression-in probability are different in the two experiments. The discrepancy between these experiments reflects that the misidentifyreanalyse procedure was more likely to happen in Experiment 1 than in Experiment 2. In other words, in contrast to lower frequency neighbors, it was more likely to misidentify targets as higher frequency neighbors. This suggests that, though along with Johnson (2009) and Paterson et al. (2009) we found interference effects on target processing from both higher and lower frequency neighbors, the inhibition may be manifested in reading differently based on neighbor frequency. Here we posit that some form of lateral inhibition between activated words is at the heart of the inhibitory neighbor effect. However, in the case of inhibition from higher frequency neighbors, this inhibition is strong enough that it results in a significant number of 
misidentifications during the competition. That is, the higher frequency neighbors were winning the competition on a fair number of trials. In the case of lower frequency neighbors, the inhibition was not strong enough to win the competition and therefore these lower frequency neighbors fail to yield a significant number of misidentifications (Gregg \& Inhoff, 2016).

\section{Table 8}

The results of post hoc analysis in two experiments for the first-presented trials only (Regression-in Probability context/log(Gaze duration))

\begin{tabular}{lcccc}
\hline & Estimate & SE & $\boldsymbol{z}$ & $\boldsymbol{p}$ \\
\hline Experiment 1 & & & & \\
\hline (Intercept) & 4.11 & 1.03 & 3.97 & $<.001 * * *$ \\
Context & -6.61 & 1.59 & -4.15 & $<.001^{* * *}$ \\
Context neutral:gaze & -0.84 & 0.19 & -4.53 & $<.001^{* * *}$ \\
Context biased: gaze & 0.24 & 0.22 & 1.12 & .26 \\
Experiment 2 & & & & \\
(Intercept) & 0.54 & 1.11 & 0.49 & .63 \\
Context & -2.02 & 1.89 & -1.07 & .28 \\
Context neutral:gaze & -0.24 & 0.20 & -1.22 & .22 \\
Context biased: gaze & 0.05 & 0.28 & 0.17 & .86 \\
\hline
\end{tabular}

\section{Figure 1}

The relations between GD (log) and regressions back to targets for first-presented trials 

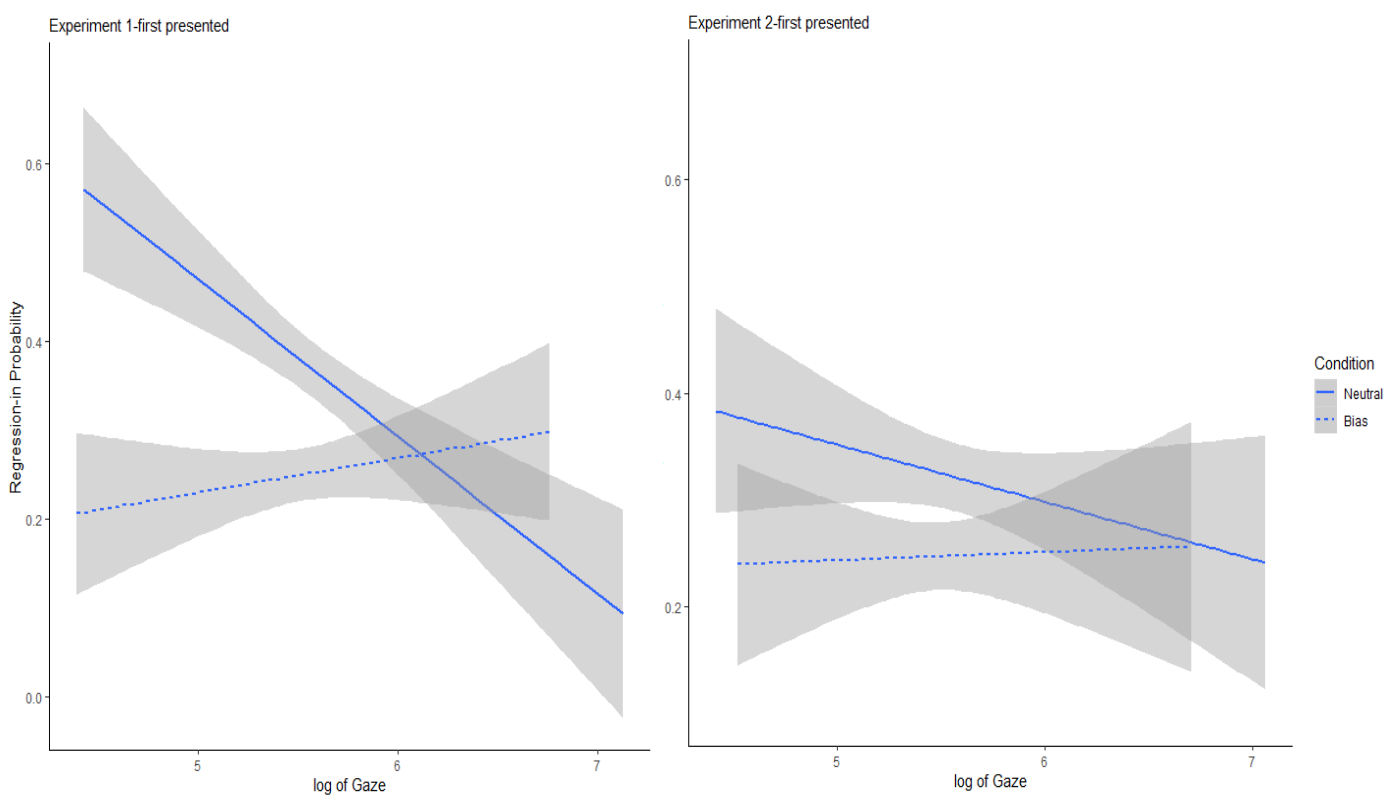

Table 9

The results of omnibus test for first-presented trials (Regression-in probability exp ${ }^{*}$ context* $\log ($ gaze $\left.)\right)$

\begin{tabular}{lcccc}
\hline & Estimate & SE & $\boldsymbol{z}$ & $\boldsymbol{p}$ \\
\hline (Intercept) & 0.18 & 0.61 & 0.30 & .76 \\
Context & 4.27 & 1.23 & 3.48 & $<.001^{* * *}$ \\
Experiment & -1.19 & 1.23 & -0.97 & .33 \\
Gaze $(\log )$ & -0.20 & 0.11 & -1.81 & .07. \\
Context:experiment & -4.71 & 2.45 & -1.92 & .05. \\
Context:gaze & -0.68 & 0.22 & -3.08 & $<.01^{* *}$ \\
Experiment:gaze & 0.19 & 0.22 & 0.87 & .38 \\
Context:gaze:exp & 0.81 & 0.44 & 1.83 & .06. \\
\hline
\end{tabular}

\section{Similarity Across Languages}

The current study investigated the context effect in real time reading in Chinese, which is structurally different from English. Despite the fact that orthographic neighbors are defined differently in English and Chinese, we found similar patterns of context effects and neighbor effects. Consistent with previous studies in English, we 
found that an increased number of plausible neighbors caused inhibition on target processing (Perea \& Pollatsek, 1998; Pollatsek et al., 1999), that context can modulate the inhibitory neighbor effects (Slattery, 2009), that both higher and lower frequency neighbors can inhibit the processing of target words (Johnson, 2009; Paterson et al., 2009), and that higher but not lower frequency neighbors are susceptible to misidentification (Gregg \& Inhoff, 2016). Based on these similarities, we speculate that the on-line use of contextual information in lexical access, the activations of orthographic neighbors, and the lateral competition among activated neighbors are general processing mechanisms independent of a specific language.

\section{Orthographic neighbors vs. Morphological families}

It should be noted that in Chinese, characters contain meanings and are morphological units. The shared character among orthographic neighbors may have different meanings. For example, the character “花” (/hua1/,flower) in “花园” (/hualyuan2/, garden) and “花店” (/hua1dian4/, flower shop) conveys a “flower” meaning, while it in “花费” (/hualfei4/, expense) and “花销” and (/hua1xiao1/, cost) conveys a “to spend” meaning. Such, the character “花” has two morphological families but only one set of orthographic neighbors. Previous studies used homographic morphemes to dissociate these two concepts (Tsang \& Chen, 2013; Zhou et al.,1999). In the current study, however, the orthographic neighbors and morphological families cannot be dissociated since we focused only on the orthographic similarities among neighbors, but not the semantic meanings of the shared characters. Future study is needed to explore whether context may influence 
the activation of orthographic neighbors and morphological families differently in Chinese reading.

Furthermore, the target words in the current study are two-character words, which can be treated as compound words. A more strict and analogous comparison between Chinese and English would be comparing the morphological family effect of two-character words in Chinese and that of compound words in English (e.g, airline, Juhasz \& Berkowitz, 2011). Future study is needed to explore the similarities and/or differences of the activation of morphological families between Chinese and English.

\section{Conclusion}

The current study found that in Chinese sentence reading, both higher and lower frequency neighbors inhibit the processing of target words, and sentence context can modulate this neighbor effect: the biased context against neighbors weakened/eliminated the inhibitory neighbor effect. Despite their different writing systems, orthographic neighbors and context influence word recognition in similar ways in Chinese and English. 


\section{Declaration}

\section{Conflicts of Interest}

The authors declared that there were no conflicts of interest with respect to the authorship or the publication of this article.

\section{Ethics Approval}

All procedures performed in studies involving human participants were in accordance with the ethical standards of the institutional and/or national research committee and with the 1964 Helsinki declaration and its later amendments or comparable ethical standards.

\section{Consent to Participate}

Informed consent was obtained from all individual participants included in the study. 


\section{References}

Acha, J., \& Perea, M. (2008). The effect of neighborhood frequency in reading:

Evidence with transposed-letter neighbors. Cognition, 108, 290-300.

Andrews, S. (1989). Frequency and neighborhood effects on lexical access:

Activation or search? Journal of Experimental Psychology: Learning, Memory and Cognition, 15(5), 802-814.

Andrews, S. (1997). The effect of orthographic similarity on lexical retrieval: Resolving neighborhood conflicts. Psychonomic Bulletin and Review, 4, 439461.

Bayen, R. H., Davidson, D. J., \& Bates, D. M. (2008). Mixed-effects modeling with crossed random effects for subjects and items. Journal of Memory and Language, 59(4), 390-412.

Barr, D. J., Levy, R., Scheepers, C., \& Tily, H. J. (2013). Random effects structure for confirmatory hypothesis testing: Keep it maximal. Journal of Memory and Language, 68(3), 255-278.

Bates, D., Kliegl, R., Vasishth, S., \& Baayen, H. (2015). https://arXiv:1506.04967

Bates, D., Mächler, M., \& Bolker, B. M. (2011). LME4: linear mixed-effects models using S4 classes. R Package Version 0.999375-39. http://CRAN.Rproject.org/package $=1 \mathrm{me} 4$

Brysbaert, M. (2019). How many participants do we have to include in properly 
powered experiments? A tutorial of power analysis with reference tables. Journal of Cognition, 2(1), 1-38.

Carreiras, M., Perea, M., \& Grainger, J. (1997). Effects of orthographic neighborhood in visual word recognition: Cross-task comparisons. Journal of Experimental Psychology: Learning, Memory, and Cognition, 23(4), 857-871.

Cole, R. A., \& Perfetti. C. A. (1980). Listening for mispronounciations in a children's story: The use of context by children and adults. Journal of Verbal Learning and Verbal Behavior, 19, 297-315.

Coltheart, M., Davelaar, E., Jonasson, J. F., \& Besner, D. (1977). Access to the internal lexicon. In S. Dornic (Ed.), Attention and performance VI (pp. 535-555). Hillsdale, NJ: Erlbaum.

Coltheart, M., Rastle, K., Perry, C., Langdon, R., \& Ziegler, J. (2001). DRC: A dual route cascaded model of visual word recognition and reading aloud. Psychological Review, 108, $204-256$.

Davis, C. J. (2003). Factors underlying masked priming effects in competitive network models of visual word recognition. In S. Kinoshita \& S. J. Lupker (Eds.), Masked priming: The state of the art. Philadelphia: Psychology Press.

Dong, J., Yang, S., \& Wang, Q. (2015). N400-like effect of stroke-based neighborhood size in Chinese characters. Journal of Neurolinguistics, 35, 120134. 
Engbert, R., Nuthmann, A., Richter, E. M., \& Reinhold, K. (2005). SWIFT: A dynamical model of saccade generation during reading. Journal of Experimental Psychology: General, 135, 12-35.

Foss, D. J., \& Blank, M. A. (1980). Identifying the speech codes. Cognitive Psychology, 12, 1-31.

Goy, H. W., Pelletier, M., Coletta, M., \& Pichora-Fuller, M. K. (2013) The effects of semantic context and the type and amount of acoustic distortion on lexical decision by younger and older adults. Journal of Speech, Language, and Hearing Research, 56, 1715-1732.

Grainger, J. (1990). Word frequency and neighborhood frequency effects in lexical decision and naming. Journal of Memory and Language, 29, 228 -244.

Grainger, J., \& Jacobs, A. (1994). A dual read-out model of word context effects in letter perception: Further investigations of the word superiority effect. Journal of Experimental Psychology: Human Perception and Performance, 20, 1158 -1176.

Grainger, J., \& Jacobs, A. M. (1996). Orthographic processing in visual word recognition: A multiple read-out model. Psychological Review, 103, 518 -565.

Grainger, J., O’Regan, J. K., Jacobs, A. M., \& Segui, J. (1989). On the role of competing word units in visual word recognition: The neighborhood frequency effect. Perception \& Psychophysics, 45, 189-195. 
Grainger, J., \& Segui, J. (1990). Neighborhood frequency effects in visual word recognition: A comparison of lexical decision and masked identification latencies. Perception and Psychophysics, 47(2), 191-198.

Gregg, J., \& Inhoff, W. A. (2016). Misperception of orthographic neighbors during silent and oral reading. Journal of Experimental Psychology: Human Perception and Performance, 42 (6), 799-820.

Huang, H. W., Lee, C. Y., Tsai, J. L., Lee, C. L., Hung, D. L., \& Tzeng, O. J. L. (2006). Orthographic neighborhood effects in reading Chinese two-character words: Reaction time and ERP measurements. Neuroreport, 17(10), 1061-1065.

Johnson, R. L. (2009). The quiet clam is quite calm: Transposed-letter neighborhood effects on eye movements during reading. Journal of Experimental Psychology: Learning, Memory, and Cognition, 35, 943-969.

Juhasz, B. J., \& Berkowitz, R. N. (2011). Effects of morphological families on English compound word recognition: A multitask investigation. Language and Cognition Processes, 26, 653-682.

Kolers, P. A., \& Ostry, D. J. (1974). Time course of loss of information regarding pattern analyzing operations. Journal of Verbal Learning \& Verbal Behavior, 13, 599-612.

Laubrock, J., Kliegl, R., \& Engbert, R. (2006). SWIFT explorations of age differences in eye movements during reading. Neuroscience and Biobehavioral Reviews, 30, 872-884. 
Li, Q. L., Bi, H. Y., Wei, T. Q., \& Chen, B. G. (2011). Orthographic neighborhood size effect in Chinese character naming: Orthographic and phonological activations. Acta Psychologica, 136, 35-31.

Li, M. F., Gao, X. Y., Chou, T. L., \& Wu, J. T. (2017). Neighborhood frequency effect in Chinese word recognition: Evidence from naming and lexical decision. The Journal of Psycholinguistic Research, 46, 227-245.

Li, X., \& Pollatsek, A. (2020). An integrated model of word processing and eyemovement control during Chinese reading. Psychological Review. Advanced online. http://dx.doi.org/10.1037/rev0000248

Marslen-Wilson, W. D. (1985). Speech shadowing and speech comprehension. Speech Communication, 4, 55-73.

McClelland, J. L., \& Rumelhart, D. E. (1981). An interactive activation model of context effects in letter perception: Part 1. An account of basic findings. Psychological Review, 88, 375-407.

Paterson, K. B., Liversedge, S. P., \& Davis, C. J. (2009). Inhibitory neighbor priming effects in eye movements during reading. Psychonomic Bulletin \& Review, 16, $43-50$.

Perea, M., \& Pollatsek, A. (1998). The effects of neighborhood frequency in reading and lexical decision. Journal of Experimental Psychology: Human Perception and Performance, 24, 767-779. 
Pollatsek, A., Perea, M., \& Binder, K. (1999). The effects of neighborhood size in reading and lexical decision. Journal of Experimental Psychology: Human Perception and Performance, 25, 1142-1158.

Pollatsek, A., Reichle, E. D., \& Rayner, K. (2006). Tests of the E-Z Reader model: Exploring the interface between cognition and eye-movement control. Cognitive Psychology, 52, 1-56.

Raney, E. G. (2003). A context-dependent representation model for explaining text repetition effects. Psychonomic Bulletin \& Review, 10 (1), 15-28.

Rayner, K., (1998). Eye movements in reading and information processing: 20 years of research. Psychological Bulletin, 124, 372-422.

Rayner, K., Ashby, J., Pollatsek, A., \& Reichle, E. D. (2004). The effects of frequency and predictability on eye fixations in reading: Implications for the E-Z Reader Model. Journal of Experimental Psychology: Human Perception and Performance, 30, 720-732.

Rayner, K., \& Duffy, S. A. (1986). Lexical complexity and fixation times in reading: effects of word frequency, verb complexity, and lexical ambiguity. Memory \& Cognition, 14, 191-201.

Rayner, K., Warren, T., Juhasz, B., \& Liversedge, S. (2004). The effects of plausibility on eye movements in reading. Journal of Experimental Psychology: Learning, Memory and Cognition, 30, 1290-1301. 
Reichle, E. D., Pollatsek, A., Fisher, D. L., \& Rayner, K. (1998). Toward a model of eye movement control in reading. Psychological Review, 105, 125-157.

Reichle, E. D., Pollatsek, A., \& Rayner, K. (2007). Modeling the effects of lexical ambiguity on eye movements during reading. In R. van Gompel, M. Fischer, W. Murray, \& R. Hill (Eds.), Eye movements: A window on the mind and brain. Amsterdam: Elsevier Science.

Reichle, E. D., Rayner, K., \& Pollatsek, A. (1999). Eye movement control in reading: Accounting for initial fixation locations and refixations within the E-Z Reader model. Vision Research, 39, 4403-4411.

Reichle, E. D., Warren, T., \& McConnell, K. (2009). Using EZ Reader to model the effects of higher level language processing on eye movements during reading. Psychonomic Bulletin \& Review, 16(1), 1-21.

Reilly, R., \& Radach, R. (2003). Foundations of an interactive activation model of eye movement control in reading. In J. Hyona, R. Radach, \& H. Deubel (eds.), The mind's eye: Cognitive and applied aspects of eye movements research (429-456). Oxford: Elsevier.

Reilly, R., \& Radach, R. (2006). Cognitive Systems Research, 7, 34-55.

Richter, E. M., Engbert, R., \& Kliegl, R. (2006). Current advances in SWIFT. Cognitive Systems Research, 7, 23-33. 
Rumelhart, D. E., \& McClelland, J. L. (1982). An interactive activation model of context effects in letter perception: Part 2. The contextual enhancement effect and some tests and extensions of the model. Psychological Review, 89, 60 -94.

Sears, C. R., Campbell, C. R., \& Lupker, S. J. (2006). Is there a neighborhood frequency effect in English? Evidence from reading and lexical decision. Journal of Experimental Psychology: Human Perception and Performance, 32, 1040 1062.

Scarborough, D. L., Cortese, C., \& Scarborough, H. S. (1977). Frequency and repetition effects in lexical memory. Journal of Experimental Psychology: Human Perception \& Performance, 3, 1-17.

Schad, D. J., Vasishth, S., Hohenstein, S., \& Kliegl, R. (2020). How to capitalize on a priori contrasts in linear (mixed) models: A tutorial. Journal of Memory and Language, 110, 104038.

Slattery, T. J. (2009). Word misperception, the neighbor frequency effect, and the role of sentence context: Evidence from eye movements. Journal of Experimental Psychology: Human Perception and Performance, 35, 1969 -1975.

Snell, J., van Leipsig, S., Grainger, J., \& Meeter, M. (2018). OB1-reader: A model of word recognition and eye movements in text reading. Psychological Review, 125(6), 969-984. 
Staub, A., White, S. J., Drieghe, D., Hollway, E. C., \& Rayner, K. (2010). Distributional effects of word frequency on eye fixation durations. Journal of Experimental Psychology: Human Perception and Performance, 36, 1280-1293.

Tsai, J. L., Lee, C. Y., Lin, Y. C., Tzeng, O. J. L., \& Hung, D. L. (2006). Neighborhood size effects of Chinese words in lexical decision and reading. Language and Linguistics, 7(3), 659-675.

Tsang, Y. K., \& Chen, H. C. (2013). Early morphological processing is sensitive to morphemic meanings: Evidence from processing ambiguous morphemes. Journal of Memory and Language, 68, 223-239.

Wang, J., Tian, J., Han, W., Liversedge, S. P., \& Paterson, K. B. (2014). Inhibitory stroke neighbour priming in character recognition and reading in Chinese. The Quarterly Journal of Experimental Psychology, 67(11), 2149-2171.

Warren, T., \& McConnell, K. (2007). Investigating effects of selectional restriction violations and plausibility violation severity on eye-movements in reading. Psychonomic Bulletin \& Review, 14, 770-775.

Wu, J. T., \& Chen, H. C. (2003). Chinese orthographic priming in lexical decision and naming. Chinese Journal of Psychology, 45, 75-95.

Williams, C. C., Perea, M., Pollatsek, A., \& Rayner, K. (2006). Previewing the neighborhood: The role of orthographic neighbors as parafoveal previews in reading. Journal of Experimental Psychology: Human Perception and Performance, 32 (4), 1072-1082. 
Yang, F. L., \& Wu, J. T. (2014). Orthographic inhibition between characters with identical semantic radicals in primed character decision tasks. Chinese Journal of Psychology, 56, $49-63$.

Zhou, X., Marslen-Wilson, W. D., Taft, M., \& Shu, H. (1999). Morphology, Orthography, and Phonology in Reading Chinese Compound Words. Language and Cognitive Processes, 14, 525-565. 